\title{
Experiences with Radioisotope Renography in Children
}

\author{
J. H. JOHNSTON and IRENE M. IRVING \\ From Alder Hey and Royal Liverpool Children's Hospitals, and the Department of \\ Paediatric Urology, University of Liverpool
}

Radioisotope renography provides, from the individual kidney, a graphic record of the renal vascularity, of the excretory funtion of the tubular cells, and of the evacuatory capability of the tubules and the kidney pelvis. By the use of additional scintillation counters, an index of the rate of extraction of the isotope from the blood and its rate of accumulation in the bladder can also be obtained.

Since its original description in 1956 by Taplin, Meredith, Kade, and Winter, renography has been extensively employed in the investigation of renal disease in adults. Only one contribution relating to its use in children has previously been made in an English language publication and in that series the investigation was limited to children over the age of 3 years (Wenzl, Tauxe, Burke, Hunt, and Stickler, 1965a, b).

The present article is an analysis of 207 renographic examinations performed in 159 children, ranging in age from 1 week to 15 years, with various types and degrees of renal pathology, mostly of a surgical nature. The main object of performing the test was to obtain an assessment of renal function and emptying ability which, by repetition, could detect changes in the pathological state of the individual kidney. At the start of the investigation it was not known whether or not valid conclusions could be drawn from repeated renograms and the question of the demonstration of alterations in renal pathology is not discussed here though many of the repeat examinations are included in the analysis. Renograms performed after the relief of obstructive uropathy are not included.

\section{Technique}

${ }^{131}$ I 'hippuran' was injected intravenously in a dosage of $0.5 \mu \mathrm{c} / \mathrm{kg}$. One counter was directed at each kidney and a third was applied either to the chest or to the lower abdomen over the bladder according to whether interest was focused on the clearance of isotope from the blood or on its rate of evacuation from the upper urinary tract. A meter range of 100 was used in

Received February 3, 1967. most children, but in infants, in whom the isotope dosage was small, a range of 30 was necessary. The time constant was 10 seconds in all cases and at all phases of the tracings. For the kidney tracings a paper speed of $1.25 \mathrm{~cm}$./min. was used; for the tracings from the bladder or the chest the paper speed was 15 $\mathrm{cm}$./min. and the recordings here were made for brief periods intermittently. (The tracings illustrated in the Figures have been extrapolated to a paper speed of half that of the originals in order to facilitate publication.) With a pathological kidney the examination lasted at least 20 minutes; normal tracings were often completed in a shorter period. In the great majority of instances renography was carried out with the child in the supine position. No preliminary preparation was employed and the children were in a normal state of hydration.

The tracings fall into two groups; those from children over 2 years of age and those from children under 2 years.

\section{Tracings from Children Over 2 Years}

(1) Tracings from normal kidneys. The normal renogram at this age is the same as that seen in the adult, with three well-defined phases (Fig. 1). The first, 'the vascular spike', begins a few seconds after the injection; radioactivity rises rapidly and reaches a peak within 30 to 60 seconds. The height of this segment is representative of the volume of the blood flow to the kidney, but a certain proportion of it derives from irradiation from non-renal tissues. The second or 'functional' segment is a slower rise to an apex of radioactivity and indicates the rate of uptake of the isotope 'hippuran' by the renal tubular cells; the total height of this segment and its speed of ascent are an index of tubular function. With the third or 'evacuatory' phase the tracing falls, at first rapidly and then more slowly, until a plateau is reached; the speed of descent indicates the rate of emptying of the kidney. During the second and third phases the background irradiation, for example from the chest, falls as the isotope is extracted from the blood by the kidneys. A rise in radioactivity over the bladder coincides with the beginning of the renal evacuatory phase. Although the segments of the normal renogram are quite distinct from each other, the processes they 


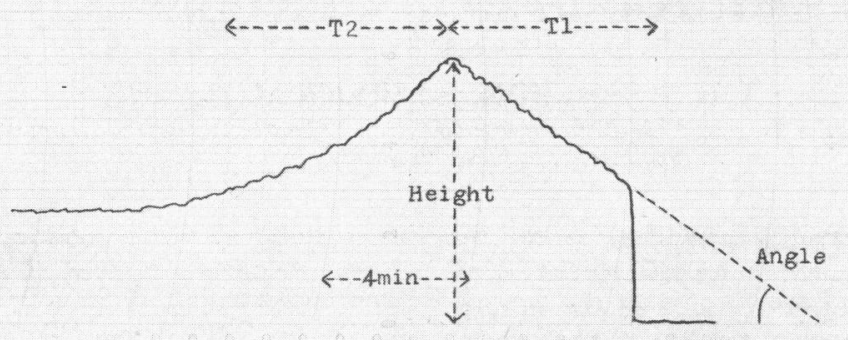

Fig. 1.-Normal renogram tracing in child over 2 years old showing the three phases (from right to left) and indicating the parameters measured. (This tracing and those in Fig. 2, 3,4,5,6, and 7 have been extrapolated from the originals to a paper speed of $0.6 \mathrm{~cm} . / \mathrm{min}$. in order to facilitate publication.)

represent overlap. Klapproth, Hirakawa, and Corcoran (1962) have shown, from dog experiments, that tubular cell accumulation of isotope occurs during the first phase and that much of the isotope is already in the lumen of the tubules in the second phase. These points must be kept in mind in the interpretation of abnormal tracings.

In order to determine the limits of normality, the four parameters indicated in Fig. 1 were measured from tracings from 57 normal kidneys (Table I).

(a) $\mathrm{T} 1$ is the time interval between the initial rise in radioactivity after the injection and the apex of radioactivity at the end of the second phase.

(b) T2 is the time required for the tracing to fall from this peak level to half that height during the evacuation phase.

(c) The 'height' is the maximal height of the tracing at a meter range of 100 , expressed as a percentage of the dosage of isotope in microcuries $(\mu \mathrm{C})$.

These three parameters are comparable to those used by Krogsgaard and Friis (1964).

(d) The 'angle' is the slope of the second segment of the tracing to the horizontal; this parameter was found by Dawborn and Doyle (1963) to be the feature of the renogram which best reflected renal function. In most instances the line of the second segment was obvious and the angle was readily measured, but in some the first segment merged gradually into the second so that the junction between them was not clearly defined, and/or the transition from the second segment to the third segment took the form of a gentle curve rather than a peak. In these cases the angle was measured between the maximal slope of the second segment and the horizontal. A few tracings from normal kidneys had to be excluded from the analysis because the maximal slope of the second segment could not be estimated with reasonable accuracy.

The normal kidneys listed in Table I were divided into four groups (graded 1-4) according to the function of the opposite kidney. Grade 1 corresponded to normal contralateral function, grade 4 was complete absence of function, and grades 2 and 3 were intermediate. $\mathrm{T} 1$ and $\mathrm{T} 2$ showed wide variation, the extremes being $2 \frac{1}{2}$ and 10 minutes and 2 and $16 \frac{1}{4}$ minutes, respectively. The height and the angle also varied widely; as would be expected, however, the levels for the

TABLE I

Analysis of Tracings from Normal Kidneys in Children over 2 Years

\begin{tabular}{|c|c|c|c|c|c|c|c|c|c|}
\hline \multirow{2}{*}{$\begin{array}{l}\text { No. of } \\
\text { Kidneys }\end{array}$} & \multirow{2}{*}{$\begin{array}{l}\text { Opposite } \\
\text { Kidney }\end{array}$} & \multicolumn{2}{|c|}{ T1 (min.) } & \multicolumn{2}{|c|}{$\mathrm{T} 2$ (min.) } & \multicolumn{2}{|c|}{ Height (\%) } & \multicolumn{2}{|c|}{ Angle $\left({ }^{\circ}\right)$} \\
\hline & & Range & Average & Range & Average & Range & Average & Range & Average \\
\hline $\begin{array}{r}18 \\
13 \\
20 \\
6\end{array}$ & $\begin{array}{l}\text { Grade } 1 \\
\text { Grade } 2 \\
\text { Grade } 3 \\
\text { Grade } 4\end{array}$ & $\begin{array}{c}3-9 \\
2 \cdot 5-9 \\
2 \cdot 5-10 \\
2 \cdot 5-6\end{array}$ & $\begin{array}{l}5 \cdot 9 \\
5 \cdot 3 \\
4 \cdot 8 \\
4 \cdot 5\end{array}$ & $\begin{array}{c}2 \cdot 2-15 \cdot 5 \\
5-16 \cdot 2 \\
2-16 \\
3 \cdot 2-8 \cdot 2\end{array}$ & $\begin{array}{l}8 \cdot 0 \\
8 \cdot 4 \\
7 \cdot 3 \\
5 \cdot 7\end{array}$ & $\begin{array}{c}31-95 \\
34-116 \\
43-131 \\
56-114\end{array}$ & $\begin{array}{c}60 \cdot 2 \\
65 \cdot 5 \\
76 \\
82 \cdot 8\end{array}$ & $\begin{array}{l}11-34 \\
12-52 \\
18-53 \\
26-52\end{array}$ & $\begin{array}{c}19 \cdot 3 \\
26 \cdot 8 \\
35 \cdot 5 \\
34\end{array}$ \\
\hline
\end{tabular}




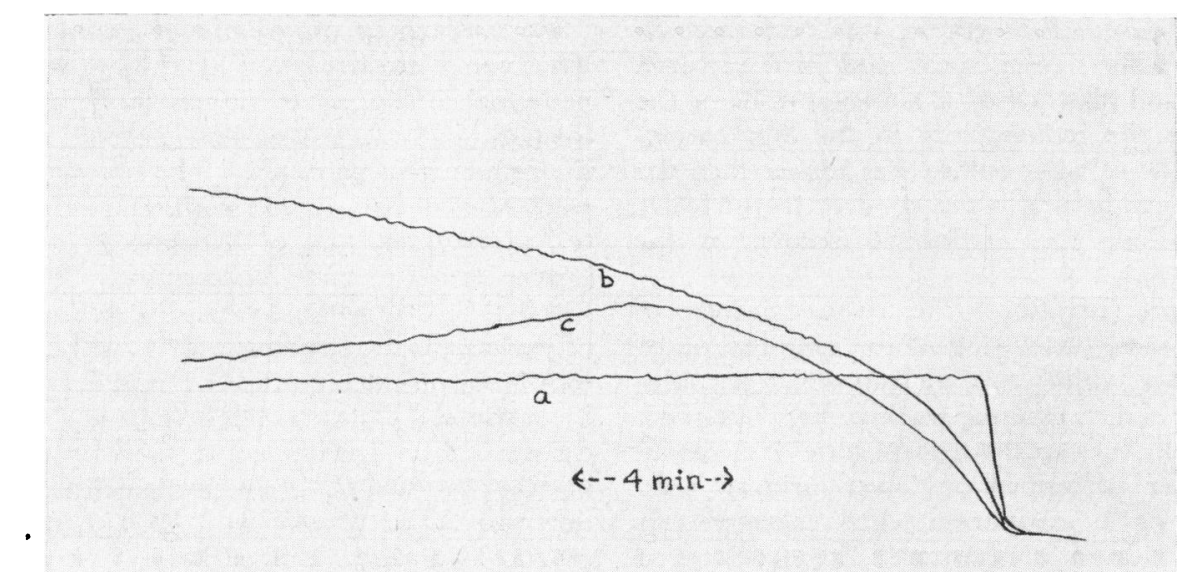

FIG. 2.-Abnormal tracing from pathological kidneys in children over 2 years old. a. Flat tracing. b. Rising tracing. c. Subnormal tracing.

normal kidneys were dependent on the severity of disease in the opposite organ, being greatest when the latter showed marked impairment or absence of function. Attempts to combine all four parameters into a formula ending with one final figure produced such extreme variations as to be quite valueless. Our findings endorse the contention of Winter (1963) that the renogram should be regarded as a qualitative rather than a quantitative test of renal functional capacity and that the important feature is the over-all shape of the tracing rather than the measurement of its individual components.

The question of the reproducibility of the renogram is of great significance in regard to the possibility of using the test repeatedly as an index of changes in the pathological status of the kidney. Some authors (Dawborn and Doyle, 1963) have found that there is indifferent agreement between repeated tracings from the same kidney. In our series, 8 children over 2 years of age, each with one normal and one pathological kidney, underwent two renographic examinations. The shortest interval between them was 6 months. In one child the two tracings from the normal kidney were virtually identical. In the others, the heights relative to the dosage and the slopes of the second segments tallied remarkably closely, considering the wide variations of the normal, and the differences between them could be explained by alterations in the function of the opposite pathological kidneys. T2 from the normal kidneys was less reproducible, but in each case the tracing from the opposite kidney varied in the same direction as the normal one, indicating that extrarenal influences were responsible for the production of the altered parameter in successive tracings.
2. Abnormal tracings from pathological kidneys. Three types of abnormal tracing were recognizable: the flat, the rising, and the subnormal (Fig. 2a, $b$, and $c$ ).

Flat tracing (Fig. 2a). With this type there is no second phase; after the initial upsweep the tracing either continues parallel to the horizontal, or, after a short plateau, falls gradually. The appearance implies either a failure of isotope 'hippuran' to reach the tubular cells or that the cells extract the isotope from the blood very slowly, if at all, and is an indication of severe impairment of renal function. Flat tracings (Fig. 2a) were obtained from 52 kidneys of varied pathology in the series, 21 with a dilated upper urinary tract, 14 of which were due to obstruction and 7 associated with reflux, and 31 with an undilated urinary tract but with parenchymal disease such as dysplasia, pyelonephritis, posttraumatic atrophy, tuberculosis, nephrocalcinosis, and glomerulonephritis. Intravenous pyelography on the 52 kidneys showed no concentration in 29; 23 were visible on pyelography but the concentration was impaired and/or the kidney was considerably diminished in size. In 3 children with bilateral flat tracings and with only one kidney visible on pyelography, the tracing from the radiologically visible kidney was significantly higher than the other, which suggests that bilateral flat tracings of unequal height are of significance in the assessment of the functional capacity of one kidney relative to the other.

No conclusions could be drawn concerning renal function by comparing the height of a flat renal tracing and the height of the background irradiation. Indeed, a flat loin tracing of greater height than the background does not even indicate that a functioning 
kidney is present. Renography was performed in 11 children after nephrectomy and in 2 children with congenital absence of a kidney; in 10 of the examinations the radioactivity in the loin corresponding to the missing kidney was higher than the background irradiation in the chest or in the lower abdomen before the arrival of isotope in the bladder.

Rising tracing (Fig. 2b). This either continues to rise slowly to the conclusion of the examination or else rises more rapidly to a plateau which is maintained to the end. 33 rising tracings were obtained. All these kidneys were hydronephrotic as a consequence either of upper or lower urinary tract obstruction, or in association with vesico-ureteric reflux. All kidneys concentrated the medium on intravenous pyelography.

Subnormal tracing (Fig. 2c). In these the three phases of the normal renogram are present but the tracing lies outside the normal limits as regards the slope of the second segment, $\mathrm{T} 1, \mathrm{~T} 2$, or all these three parameters.

Tracings from 27 kidneys were of this type. 13 were hydronephrotic, 12 as a consequence of obstruction, and 1 in association with reflux; 12 of the 13 were visible on pyelography.

In these hydronephrotic kidneys, the abnormality of the tracing was restricted to the evacuatory phase in 6 ; all phases were abnormal in 7 .

Thirteen kidneys had pyelonephritic scarring on pyelography, with or without vesico-ureteric reflux; in 11 of these, all phases of the tracings were abnormal, and in 2 only the third phase was abnormal.

One kidney was associated with reflux but showed no scarring on pyelography.

Abnormal delay in the evacuation phase with an otherwise normal tracing is therefore suggestive but not conclusive of upper urinary tract dilatation. In 3 children with bilateral subnormal tracings of unequal height the higher tracing was, in each case, obtained from the kidney which showed the better function on pyelography.
Renogram in diagnosis of renal pathology. The type of tracing given by kidneys with different pathological lesions is shown in Table II. The dilated unobstructed ureters were all examples of the refluxing megaureter. The obstructed kidneys were cases of pelvi-ureteric obstruction, of obstructed megaureter, and of hydroureter secondary to lower urinary tract obstruction. The kidneys listed as having parenchymal disease included cases of pyelonephritic scarring with or without reflux, of dysplasia, of calculus disease, and of renal damage by trauma, glomerulonephritis, and nephrocalcinosis. It is apparent that there is considerable overlap between different pathological lesions and different types of tracing. Rising tracings were obtained only from dilated kidneys showing pyelographic function, but renal dilatation with impaired function may produce a flat tracing or, if the obstruction is less severe, a subnormal tracing with a delayed evacuating phase. Parenchymal disease with pyelographic concentration commonly gave a subnormal or a normal tracing; kidneys without pyelographic function or with considerable diminution in size nearly always produced a flat tracing.

Fifteen undoubtedly pathological kidneys in the series gave tracings that were within the normal range; the 3 obstructed kidneys were, however, only mildly to moderately dilated, and 11 of the 12 cases of parenchymal disease showed only localized pyelonephritic scarring, mostly associated with reflux. The remaining kidney contained a calculus in the pelvis. 11 of these 15 pathological kidneys were associated with a normal opposite kidney. In 8 instances the tracing from the pathological kidney, though within the normal range, was of lower height than that from the normal kidney; in two cases the tracing from the pathological organ was the same height as that from the normal but the tracing was inferior in at least one other parameter. In only one instance, the child with a renal pelvic calculus, was the tracing from the abnormal kidney the equal of that from the normal.

TABLE II

Type of Tracing given by Pathological Kidneys before Operation in Children over 2 Years

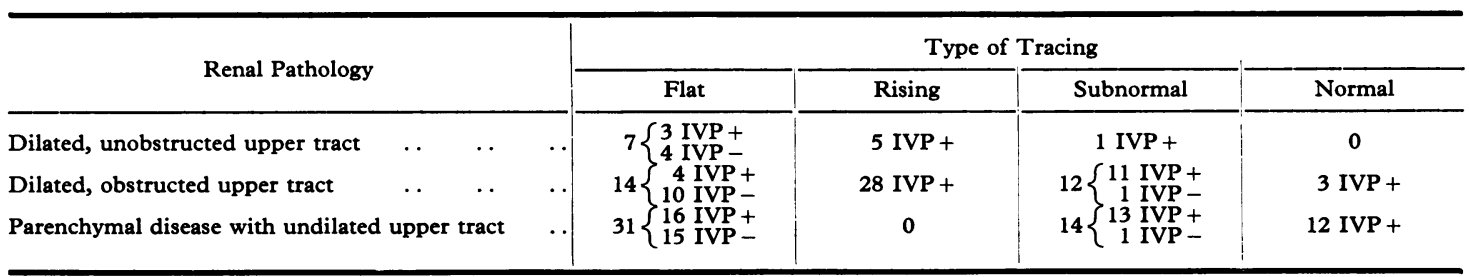

IVP $+=$ radiological concentration 


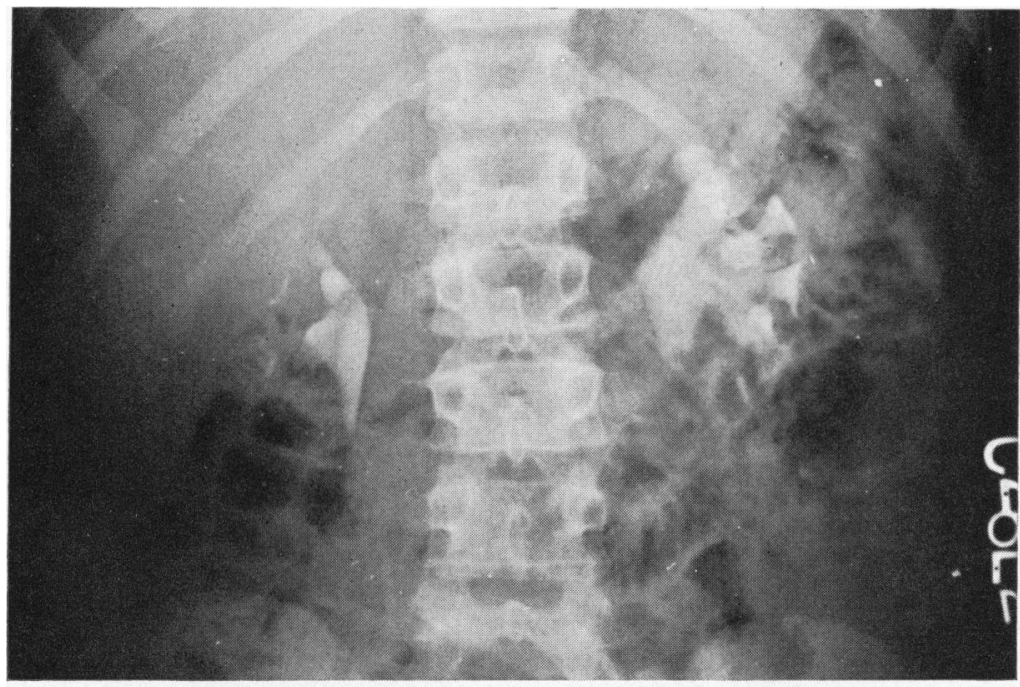

(a)

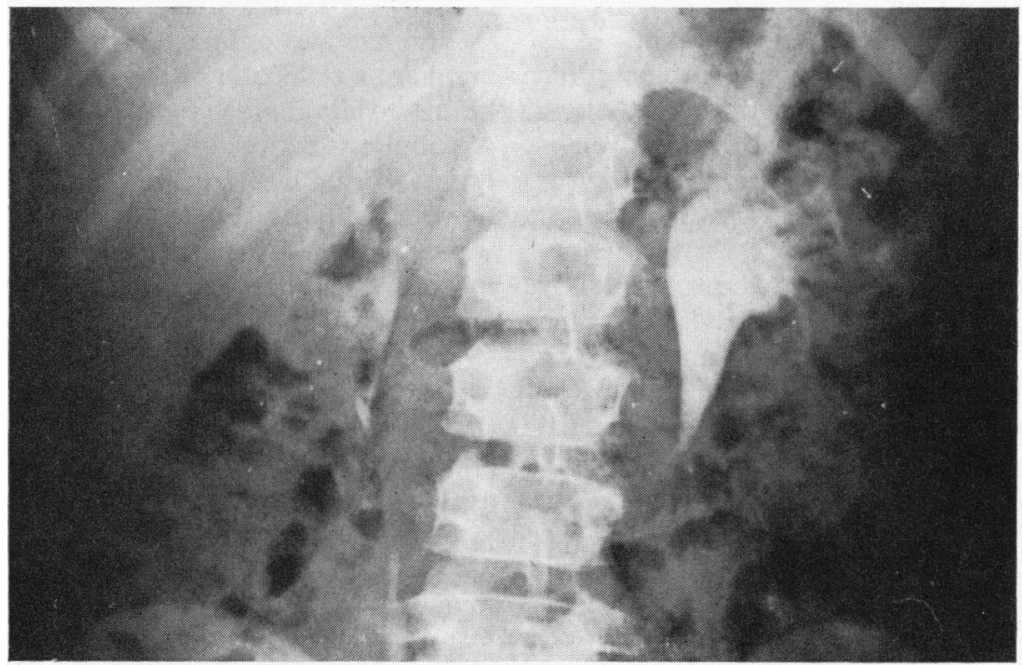

(b)

Fig. 3.-Intravenous pyelogram of a boy aged 11 years, with left loin pain. (a) 15 minutes' exposure. Slight pelvic and calyceal dilatation in left kidney. (b) 25 minutes' exposure. Left kidney slow to empty. Funnelled pelvi-ureteric junction.

Tracings within the normal range were also obtained from 24 kidneys which were doubtfully pathological. These included kidneys with slight pyelographic abnormality of uncertain nature, kidneys associated with ureteric reflux but showing normal pyelograms and cases of ureteric duplication, of haematuria of unknown causation, and of urethral obstruction with an undilated upper urinary tract.

In only one child in the series did renography give information of immediate therapeutic importance which was not obtained by the standard methods of urological investigation. S.E., a boy aged 11 years, had complained of intermittent left loin pain for some months. Intravenous pyelography (Fig. 3) showed slight calyceal clubbing on the left side and delay in emptying of the left kidney. There was, however, no dilatation of the renal pelvis and no suggestion of pelvi-ureteric 


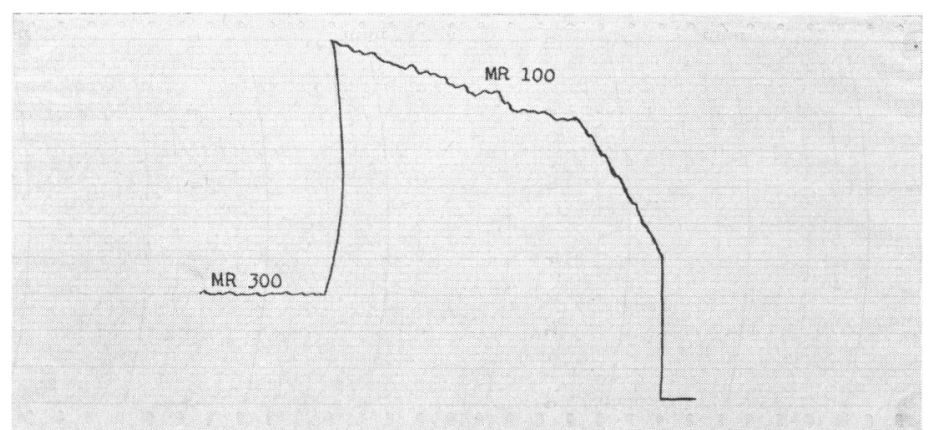

FIG. 4.-Renogram of left kidney in the same boy as in Fig. 3. Rapidly rising tracing indicates severe stasis in association with rapid excretion of isotope.

obstruction. Retrograde pyelography showed a similar appearance and was not helpful. Renography gave a normal tracing on the right; on the left, the tracing was of the rapidly rising type, indicative of severe urinary stasis in a kidney with virtually normal function (Fig. 4). At operation, an aberrant artery and vein were found to cross the ureter just below the pelvi-ureteric junction. The apparent contradiction between the pyelographic and the renographic interpretations of the pathology probably resulted from the fact that during pyelography the boy was dehydrated and, though there was some delay in emptying, there was insufficient urinary flow to distend the pelvis. During renography, on the other hand, the patient was fully hydrated and it is likely that the pelvis was then distended to a sufficient degree to angulate the ureter over the aberrant vessels, as was seen to occur at operation when saline was injected into the renal pelvis.

\section{Tracings from Children Under 2 Years}

(1) Tracings from normal kidneys. Eleven tracings were obtained from normal kidneys in children under the age of 6 months. 9 of them were flat, without any rise of radioactivity after the initial rise; 2 tracings, from one child, showed a slight second phase rise, the angles to the horizontal being $8^{\circ}$ and $7^{\circ}$. 6 tracings from children aged 7 to 21 months were subnormal or minimally normal by the standards of the older child; the average angle of the second segment was $13^{\circ}$, the range being $10^{\circ}$ to $18^{\circ}$. T 1 and $\mathrm{T} 2$ were within the normal range for the child over 2 years of age.

The flat and subnormal tracings of early childhood are a reflection of the impaired capacity of the kidney to eliminate 'hippuran' because of immaturity of tubular function. Vesterdal and Tudvad (1949) estimated the clearance of para-amino-hippurate in newborn infants to be one-tenth that of the adult when corrected for surface area; in the first three months of life the clearance reached only one-half the adult value.

(2) From pathological kidneys. The types of tracings obtained from 49 pathological kidneys in children under 2 years old are shown in Table III. The kidneys listed as showing renal atrophy were mostly dysplastic organs; in some, superimposed pyelonephritis was significant in causing the parenchymal disease. The tracings classified as 'subnormal' had parameters below the normal standards of the child over 2 years old. The flat tracings given by the pathological kidneys were,

TABLE III

Type of Tracing given by Pathological Kidneys Before Operation in Children under 2 Years

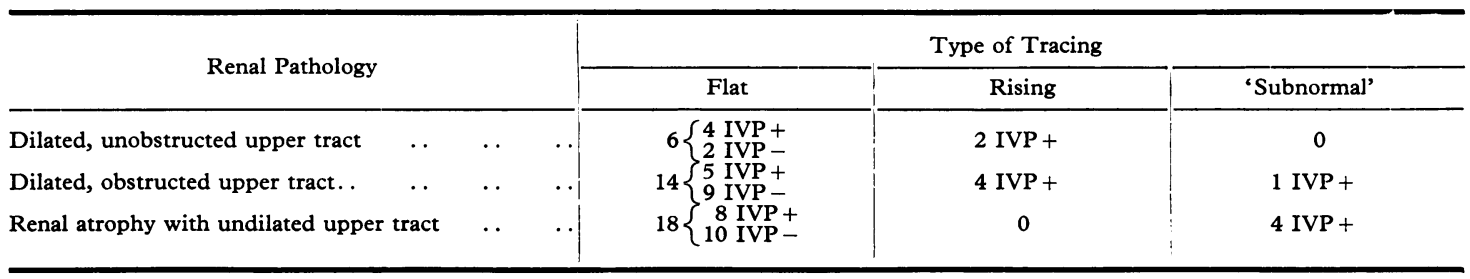

IVP $+=$ radiological concentration. 
individually, indistinguishable from those given by normal kidneys in children under 6 months of age; the heights of the tracings relative to the dosage were similar in both groups of cases.

In those infants with 2 severely diseased kidneys, however, the bladder tracing remained low, whereas when at least one kidney was normal the bladder tracing reached a level of radioactivity much higher than that of either kidney (Fig. 5 and 6). Bilateral flat tracings of unequal height were obtained in 8 patients; in each instance the higher tracing was given by the kidney which showed the better function on intravenous pyelography (Fig. 7).

\section{Discussion}

Isotope renography gives a qualitative index of the tubular function and the emptying capability of the individual kidney. The test requires no special preparation and since the radiation dosage is minute it can safely be repeated at intervals. In the child over 2 years of age the normal tracing shows the same 3 phases as in the adult. The range of normality, as judged by several parameters, is undoubtedly wide but the variations in the height of the tracing relative to the dosage and in the slope of the second segment are less marked if account is taken of the function of the opposite kidney. The renogram is also influenced by extrarenal factors. Meade and Shy (1961) found that pain and anxiety diminished the height and the slope of the tracing, presumably by influencing the renal vascular flow, and Wenzl et al. (1965a) recorded that, in children, dehydration had a pronounced effect in lengthening the tracing, particularly the excretory slope; such general influences obviously affect the tracings of both kidneys similarly. Provided allowance is made for these contralateral and extrarenal influences, the renogram for the individual normal kidney is, in our experience, reproducible to a significant degree.

It is not possible to judge from a flat tracing whether the renographic abnormality is due to poor function, non-function, or absence of the kidney. Moreover, comparing the height of the tracing with that of the background irradiation is not helpful since in several instances in our series the tracing from an empty loin was higher than the background. In addition, it is not possible to compare individual kidney function in different patients by comparing the heights of the renograms; the tracing height depends not only on the dosage of the isotope but also on the distance between the counter and the kidney which is, in turn, dependent on the thickness of the body parietes. Conclusions can, however, be drawn from tracings, flat or otherwise, of unequal height in the same child; in each instance in our

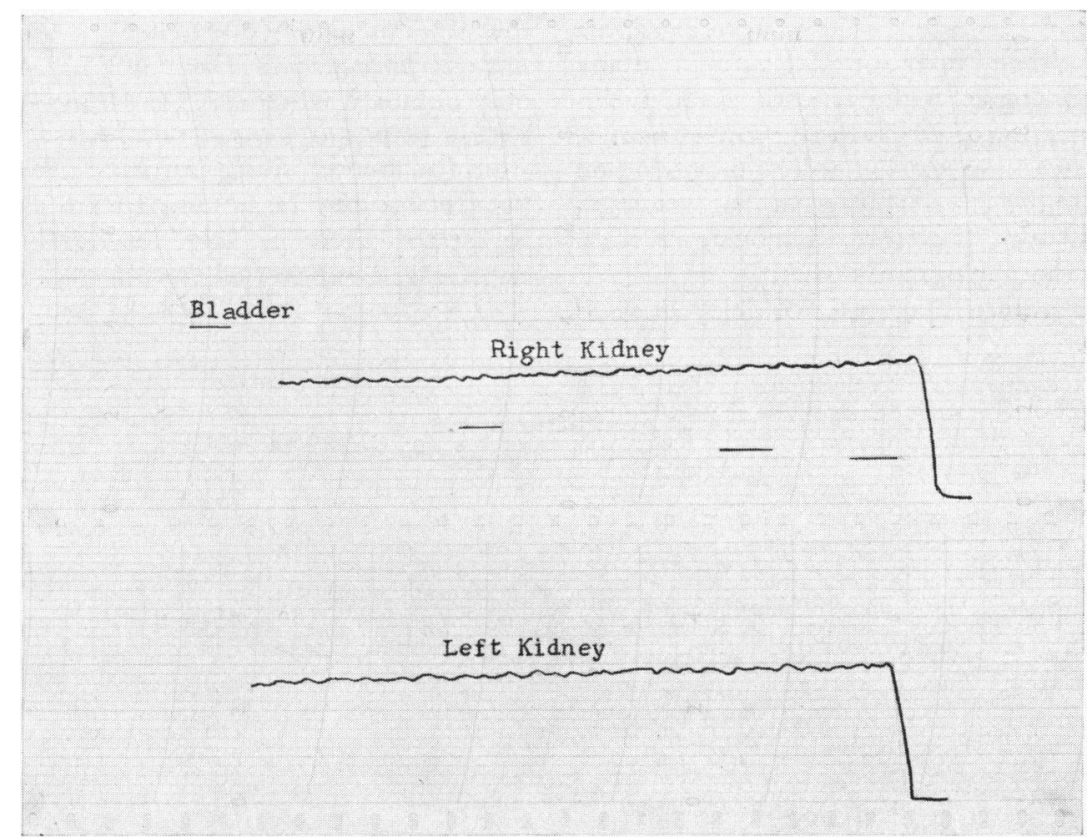

FIG. 5.-Renograms of infant aged 3 months with bilateral renal hypoplasia and blood urea of 116. Bilateral flat renal tracings. Little rise in bladder radioactivity. 


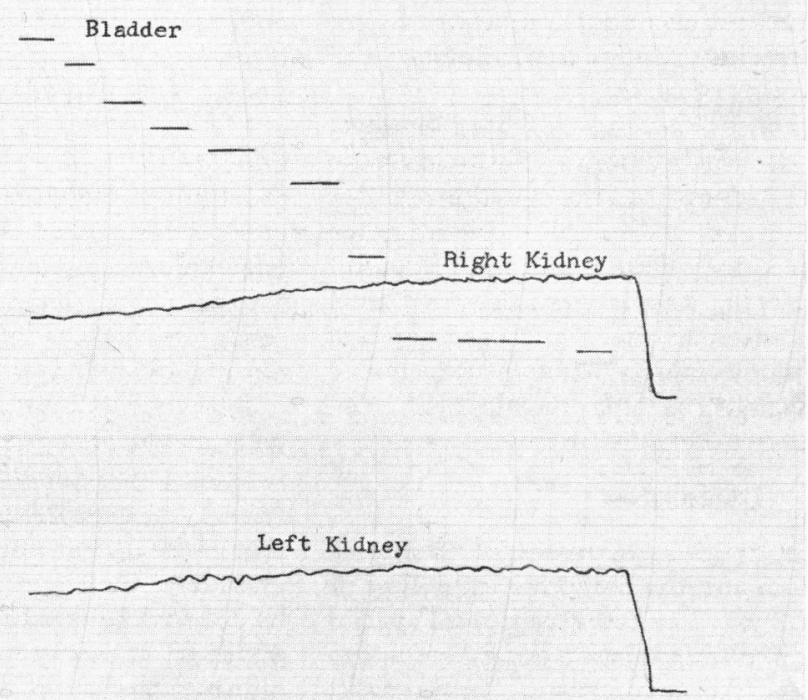

FIG. 6.-Renograms in infant aged 5 months with a normal urinary tract. Bilateral flat renal tracings. The bladder tracing rises to a greater height than in Fig. 5.

series the higher tracing coincided with the better or only functioning kidney.

The isotope renogram is a more sensitive index of defective renal function than is intravenous urography. Of 52 kidneys giving flat tracings in children over 2 years of age, 23 showed pyelographic concentration, albeit poor or delayed in many instances. Pathological kidneys with good pyelographic concentration often gave subnormal tracings. In our experience kidneys that were not visible on pyelography almost invariably gave flat tracings; renography will not, therefore, demonstrate renal function when the pyelogram is silent.

A rising renogram is diagnostic of delay in renal emptying associated with upper urinary tract dilatation. The dilatation is not necessarily the result of obstruction unless one agrees with Hutch (1958) that there is an obstructive factor in the refluxing megaureter; our own interpretation of such cases is that the evacuatory impairment is due to ineffective ureteric peristalsis. The rising tracing is, however, only obtained when delayed emptying is associated with a sufficiently rapid renal extraction of isotope from the blood. With impaired tubular function, a flat tracing may be obtained from a dilated kidney or, when there is less interference with renal emptying, a subnormal tracing may be produced.

It is apparent from Table II that, apart from the

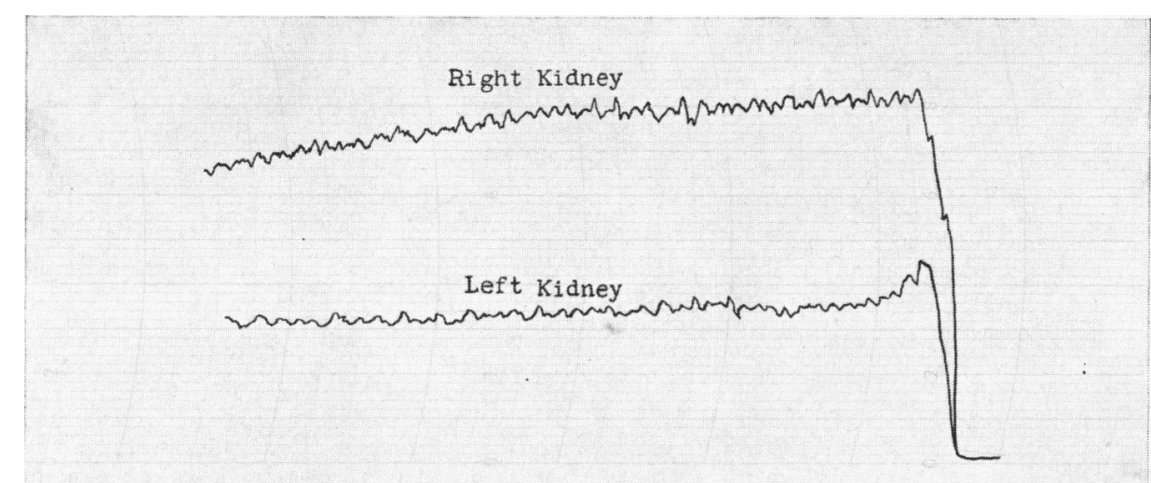

Fig. 7.-Renograms in infant aged 1 week with a normal right kidney and a large, multicystic, afunctional left kidney. Both tracings are of the flat type but that from the normal kidney is significantly higher than the other. 
deductions which can be made from a rising tracing, the type of tracing is not diagnostic of the type of renal pathology. Further, renography cannot be relied upon as an infallible screening test for renal disease, because tracings within the normal range may be obtained from obstructed kidneys with minimal dilatation or from kidneys with localized pyelonephritic scarring. When, however, the other kidney is normal, the tracing from the pathological kidney is, in nearly all instances, significantly lower in height or deficient in other parameters as compared with the normal one. In only one instance in our series were virtually identical tracings obtained from a child with one normal and one abnormal kidney; this was a 3-year-old boy with a renal calculus. Two pathological kidneys, however, may give similar tracings, each within the rather wide range of normality.

In children under 2 years of age, the fact that the normal tracing is flat or subnormal by the standards of the older child limits the value of the renogram as a test of renal function; normal and severely pathological kidneys can give precisely similar tracings. Flat tracings of unequal height in the same patient are, however, as in the older child, significant in that the higher tracing is always obtained from the better functioning kidney. An index of over-all renal function in the infant can be obtained from the bladder tracing; this remains low when the function of both kidneys is impaired but rises to a height much greater than that of either kidney tracing if at least one kidney is of normal function. When renal function is sufficient, urinary stasis and dilatation of the upper urinary tract can give a rising tracing in the infant, but not every dilated kidney with pyelographic concentration does so.

We are impressed with the value of isotope renography as a test, simple in execution and safely repeatable, of individual renal function and emptying capability. Provided such factors as changes in extrarenal influences and alterations in the function of the opposite kidney are allowed for, the renogram in the child over 2 years old is sufficiently reproducible to allow the conclusion that alterations in a tracing with the passage of time are due to alterations in renal tubular function and/or evacuatory capacity. This is particularly so in cases of unilateral disease, when it can be established that the function of one kidney remains stable. The detection of alterations in the pathological state of the individual kidney by repeat examinations constitutes the main value of renography in children and allows deductions to be made concerning the value or otherwise of therapeutic measures.

\section{Summary}

Experiences with isotope renography in 159 children are described. In children over 2 years old the normal renogram is the same as in the adult. The variations of the normal are wide and the tracing is influenced by the function of the opposite kidney and by extrarenal factors. Three types of abnormal tracing, the flat, the rising, and the subnormal are recognized. In younger children, particularly in infants under 6 months, the normal tracing is of the flat type. It is concluded that, provided allowance is made for extrarenal and contralateral influences, changes in the shape of a renogram with the passage of time are indicative of changes in the tubular function and in the evacuatory capacity of the kidney under investigation. The detection of such changes in the individual kidney constitutes the main value of renography in childhood.

We are grateful to Mr. E. C. Edwards who drew our attention to the potentialities of isotope renography in paediatric urology, to Mr. J. Cosbie Ross for permission to use the apparatus in the Liverpool Regional Urological Centre, and to Dr. W. Kulke and Dr. R. Spencer for supplying the isotope 'hippuran'.

\section{REFERENCES}

Dawborn, J. K., and Doyle, A. E. (1963). An evaluation of the radioisotope renogram. Med. F. Aust., 1, 853.

Hutch, J. A. (1958). The Ureterovesical function. Berkeley, University of California Press.

Klapproth, H. J., Hirakawa, A., and Corcoran, A. C. (1962). Functional significance of the radioisotope renogram: an experimental study. $\mathcal{F}$. Urol. (Baltimore), 87, 77.

Krogsgaard, A. R., and Friis, T. (1964). Isotope nephrography with $\mathrm{I}^{131}$ 'hippuran'. I. Technique and experience in various medical renal diseases. Acta med. scand., 176, 17.

Meade, R. C., and Shy, C. M. (1961). The evaluation of individual kidney function using radioiodohippurate sodium. $f$. Urol. (Baltimore), 86, 163.

Taplin, G. V., Meredith, O. M., Jr., Kade, H., and Winter, C. C. (1956). The radioisotope renogram: an external test for individual kidney function and upper urinary tract patency. 7. Lab. clin. Med., 48, 886.

Vesterdal, J., and Tudvad, F. (1949). Studies on the kidney function in premature and full-term infants by estimation of the inulin and paraaminohippurate clearances. Acta paediat. (Uppsala), 37, 429.

Wenzl, J. E., Tauxe, W. N., Burke, E. C., Hunt, J. C., and Stickler, G. B. (1965a). Radioisotope renography in children. I. The renogram in children without renal disease. Pediatrics, 36, 120.

,,,---- and $-(1965 b)$. Radioisotope renography in children. II. Diagnosis of renal disorders. ibid., 36, 200.

Winter, C. C. (1963). Radioisotope Renography. Williams and Wilkins, Baltimore. 